\title{
Atomic Scale Analysis of Dopants in CMOS Structures by Atom Probe Tomography
}

\author{
Isabelle Martin ${ }^{1}$, Robert Estivill ${ }^{2,3,4}$, Marc Juhel ${ }^{2}$, Adeline Grenier ${ }^{3}$, Ty J. Prosa ${ }^{1}$ and David J. Larson ${ }^{1}$ \\ ${ }^{1 .}$ CAMECA Instruments, Inc., Madison, WI, 53711 USA \\ 2. STMicroelectronics, Crolles, 38926 France \\ 3. Univ. Grenoble Alpes, Grenoble, 38000 France \\ 4. Univ. de Rouen, Groupe de Physique des Matériaux, 76800 France
}

As microelectronic devices become smaller and smaller (a few nanometers), atom probe tomography (APT) becomes one of the few techniques able to provide 3D chemical information at this scale [1]. The complexity of these devices makes acceptable analysis yield a challenge because of the intimacy of disparate materials with disparate evaporation fields [2]. This study focuses on the use of test structures to avoid yield issues and provide unique analysis information about 3D dopant distributions within these simplified device structures.

Samples patterned with gate stacks including source/drain regions and containing multiple test zones were studied and are illustrated in Fig. 1. Both n-type and p-type structures are present on bulk silicon and silicon-on-oxide substrates. The gates were arsenic-doped and the source/drain regions were phosphorous doped for n-type and boron doped for p-type. Gate and source/regions were targeted separately during specimen preparation as illustrated in Fig. 2. Focused Ion Beam (FIB) annular milling was first centered laterally over the feature of interest, but the location along the 1D line structure remained uncertain. After an initial batch of specimen preparation and APT analysis feedback, the user can properly identify visual FIB image markers to allow for accurate pattern placement thereafter.

Reconstruction parameters (evaporation field, shank angle, etc. [3]), were chosen so that the dimensions of the reconstructed features matched those observed in TEM images (Fig. 1b). In this way the localized information at the nanometers scale could be accurately extracted. Dopant distributions maps around the gate and in the source/drain region are shown in Fig. 2b and 2c. The advantage of APT is that it contains 3D information, so that 1D dopant distributions can be extracted along any direction of interest to the analyst (parallel or perpendicular to the gate). Fig. 3a shows a 3D ion map centered on the source/drain, silicon/germanium region, together with composition profiles calculated along two different orientations, in depth toward the substrate (Fig. 3b) and laterally (Fig. 3c). It is clearly observed that the boron is not uniformly distributed in the source/drain with segregation to interfaces with the silicon substrate (depth) and the silicon oxide (lateral).

\section{References:}

[1] T.F. Kelly and M.K. Miller, Rev Sci Instrum 78 (2007) p. 031101.

[2] S. Jin et al, J Vac Sci Technol B 29 (2011) p. 061203.

[3] F. Vurpillot et al, Ultramicroscopy 111 (2011) p. 1286. 
(a)

\section{P-type structures}

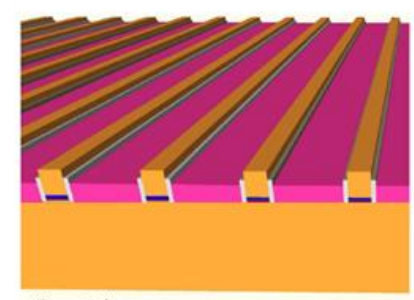

Silicon Substrate

$\mathrm{N}$-type Structures

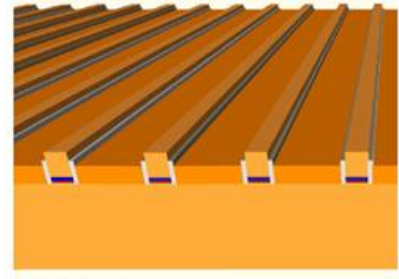

Silicon Substrate
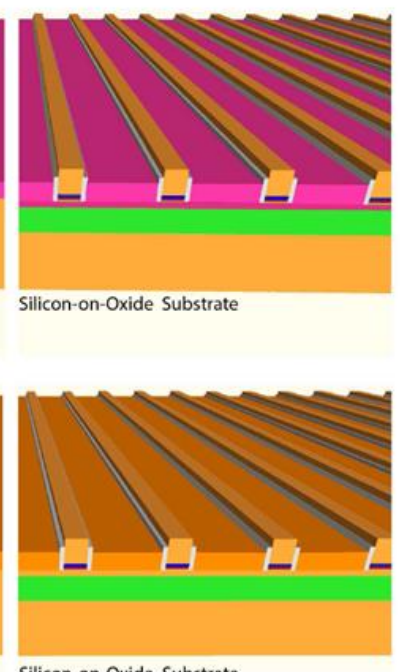

Silicon-on-Oxide Substrate (b)
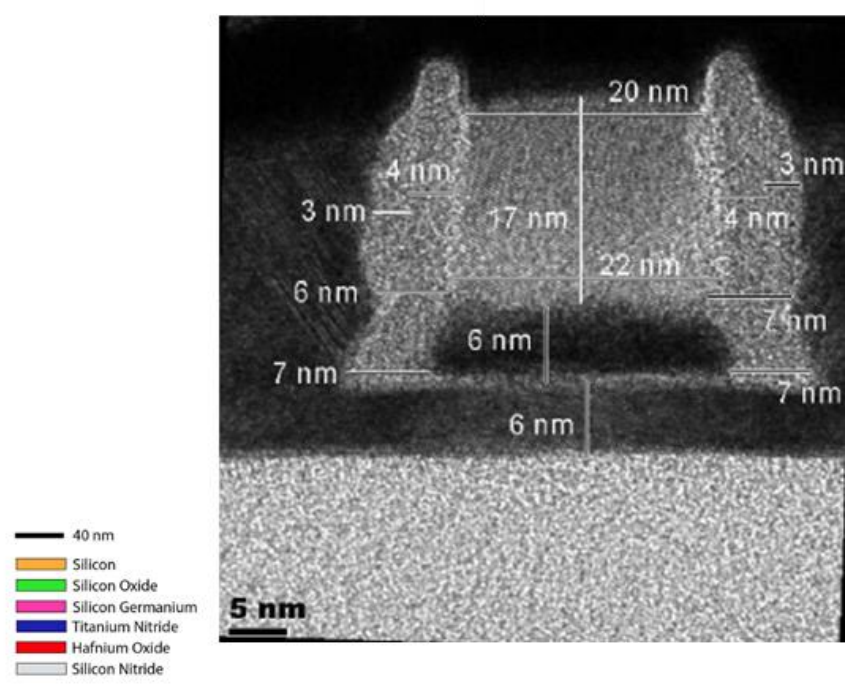

Figure 1. (a) Schematic representation of the test structures. (b) TEM micrograph of p-type device.

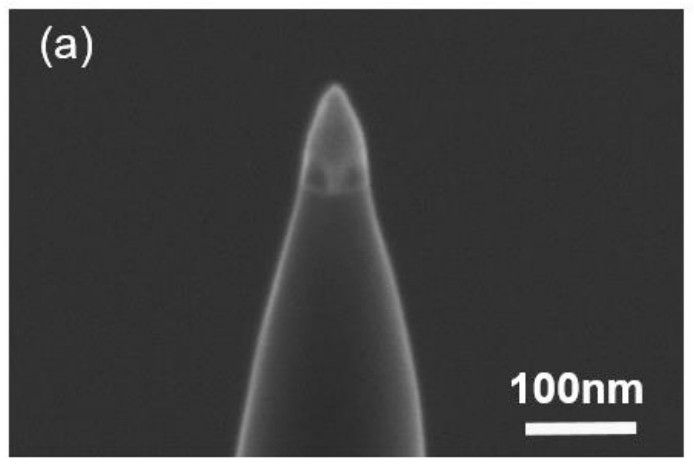

(b)

Figure 2. (a) Atom probe tip prepared by FIB. 3D ion maps of source/drain and part of the gate for (b) p-type and (c) n-type test devices.
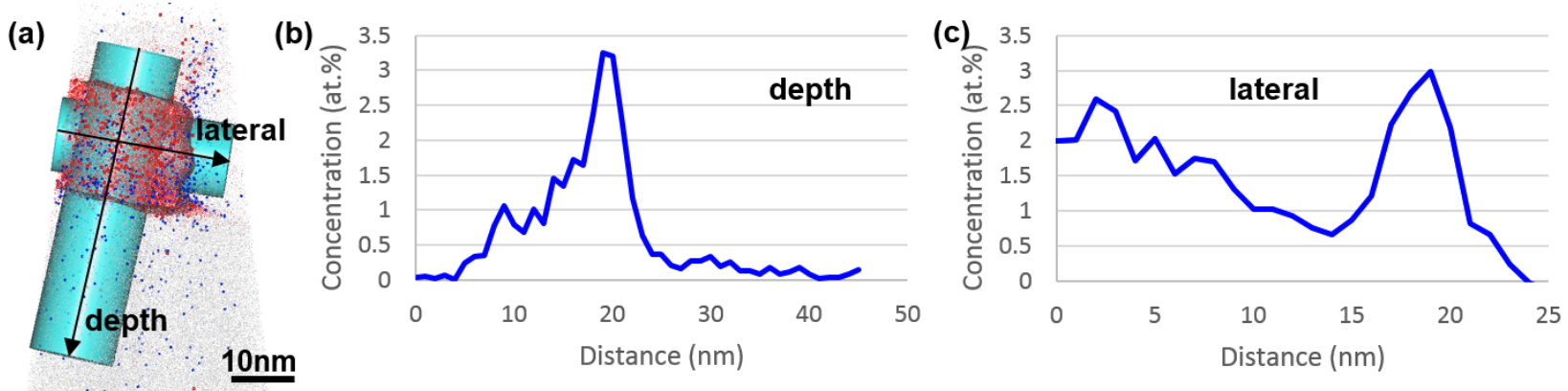

Figure 3. (a) Lateral and depth regions-of-interest used to extract Boron concentration profiles from the Silicon Germanium along the (b) depth direction and (c) lateral direction. 